\title{
Important Things to Consider In Health Information Exchange (HIE) Process
}

\author{
Yuyun Zhang ${ }^{1, a}$ \\ ${ }^{1}$ School of Nursing, Tianjin Medical University, Tianjin 300070, China. \\ alidiazhang@yahoo.com
}

Keywords: health information exchange, consolidated standards, patient engagement.

\begin{abstract}
As part of Health information technology model, Health information exchange (HIE) seek to improve health information security, integrity and interoperability, thus requires consolidated and extensive standards to guarantee the secure and convenient information storage and exchange. This paper is a literature review written after reviewing studies about the development of Internet, mobile devices and communication technology in the world of medical science; the impact of HIE on patient engagement, health behavior change, self-management, and interventions; HIE genres, current conditions, and consumer feedbacks; the important aspects to consider in the development of HIE systems including ethical issues, barriers and required standards to consider.
\end{abstract}

\section{Introduction}

Health information technology (HIT) is efficient at improving health outcomes, health care quality and self-management ability [1, 2]. Health information exchange (HIE) is part of HIT which improves provider-patient's secure and rapid access and share of information, which guarantees the completeness of patients' records so that current information and medical history can be jointly reviewed [3]. HIE also enhance patient engagement in the health care process, improve quality of care, support health care safety, and provide cost-effective health services for patients [4-7].

According to the reports about Chinese internet development conditions [8, 9], together with the development of new techniques such as mobile devices and cloud computing and big data, the internet medical chain has been formed basically. However, the development in different regions is disparate. End up to December 2015, in China, there was a significant difference in the amount of internet users between urban and rural areas with the percentage $71.6 \%$ in urban areas and only $28.4 \%$ in rural areas. The difference between provinces was also significant, with the percentage ranged from $37.4 \%$ in Yunnan to $76.5 \%$ in Beijing. Within the situation, electronic health records (EHR) were always kept in unaffiliated organizations and shared non-electronically in a delayed and non-uniform manner, thus not able to constitute complete and longitudinal health records which are required for making fitly decisions and avoiding repeated examination and inquiry. The imbalanced internet medicine development in different areas in China, the lack of widely adopted record standards, fragmented information and repeated examination have been a big problem contributing to the high cost of exchange and dangerous decision making[3, 10, 11].

Thus, developing a nationwide HIE system with the same recording, coding and transmitting standards is essential in developing an internet based health system which allows complete, private and secure information exchange between different institutions and areas in China [3]. Electronic HIE enables safer and more effective mutual use of health data (including core basic health and disease information, delivered treatments, evaluation and feedback for therapeutic schedules), effective diagnoses, faster response in emergencies, follow up with patients, longitudinal and inter-agency services and timely key public health events reports. It prevents duplicate visits, tests and information collection, allows the optimized allocation of health resources and efficient control of adverse events [3, 11-14]. Besides, it improves patients' access to multiple sources of health data and their ability of self-management, which include efficient health behavior interventions [15, 16]. 


\section{HIE Standards}

According to some reliable research [3, 17], there are three kinds of HIE-directed, query, and consumer-mediated. Directed exchange enables providers to require meaningful exchange: every provider who is legally involved in electronic health record systems can send patient health information to other providers and institutions securely. Query-based exchange is for unplanned care such as emergency measures, and allows immediate access to patients' major health problems and medical and treatment history. Consumer-mediated allows consumers to integrate, access and share their own health information, thus enhance self-management and peer group construction [18-20]. In order to make HIE more efficient, electronically automated access and share health information without the patient's assistance is the future trend.

As a complicated network which broadly support all providers and consumers in the whole country, consolidated standards and policies seek to be specified to make sure health information can be securely matched and integrated, Information requires to be consistently coded and transported in the same way so that patient data are understandable and usable to providers from different institutions [11]. Owing to the fact that personal information can be accessed and shared between health care providers and institutions, and even beyond the realm of health care for other services (such as insurance services) directly even without patients' notice or permission, it requires policies and technical infrastructures which seek to ensure patients voluntary participation, verify users identity, audit provider-patient relationships and purposes for access to certain information, ensure proper access to certain part of information, help users find certain providers and institutions, and monitor information exchange between providers and institutions [3, 21].

\section{Mobile Devices and Application for HIE}

An increased use of HIE systems in health service institutions has been combined with mobile devices and applications [22]. The explosion of applications and new mobile technologies has changed the way of communication and information share. Mobile devices are widely used for navigating a wide range of life events: communication, searching for information, sharing data and accessing digital services and online content. Thus, personal health information including health-related behavior and medical records are stored, combined and shared through mobile applications and devices [23]. Using smart phone with certain applications-such as short message service (SMS), Internet-based intervention programs and social media-can help with self-management [24-26]. According to a randomized trail [27], using phone applications to guide interview, report data, show motivational video, offer appointment for women, address potential barriers in seeking care, and handle the barriers can make an big impact on breast health promotion.

\section{Barriers and Issues to Consider}

Though HIE is used to promote human health, better HIE system does not necessarily mean higher health promotion ability. It is true that electronic health information can support medical academic learning and health improvement through research, quality improvement efforts, and predictive modeling. However, there is not yet a policy framework, public consensus, or usable technical design [21]. Besides, as health promotion requires engagement, interaction and cooperation of health service providers and audiences to exchange information and manage health conditions. Any absence of knowledge, belief, attitude or behavior, be it institutional or personal, will affect the validity of the HIE system [10, 14]. Moreover, though incorporate HIE system integrates information from different resources, incomplete or inaccurate information is still a big problem. Potential liability issues come from complicated match process for personal information, omission of patients or providers, information received from unauthentic providers, breaches in security, inappropriate uses of information, and ethical considerations [10, 11, 28, 29]. As a result, consolidated service standards, certain data codes, legal and ethical regulations are required to be established so that all information can be collected, shared and integrated with less distortion or unsafe-use. 
Consider information usage in true utilization rate, though researches [10, 21] shows that workflow will become more efficient while being revised to incorporate HIE, it might become overwhelming for each institution to manage excessive information held by large EHR databases in which information comes from several registered users. Besides, though HIE can theoretically reduce duplicate tests and information collection, it is difficult to do practically because it is hard to ensure the compatibility and interoperability of different institutions who have different recording system and diverse diagnose standards [21]. Moreover, the origin and liability of shared information is difficult to track, and the legal liability is difficult to determine if one institution use information from other institutions in case there are any errors [3]. Consequently, there is only a few parts of information that is actually shared. Certain tools and approaches help to search, filter, compare, and judge the accuracy of information about are vital for every institution and practitioner.

\section{References}

[1] S. Sawesi, M. Rashrash, K. Phalakornkule, J.S. Carpenter, J.F. Jones. The impact of information technology on patient engagement and health behavior change: a systematic review of the literature. JMIR Med Inform, 4(1), 392-403 (2016).

[2] J.D. Portz, A. Miller, B. Foster, L. Laudeman. Persuasive features in health information technology interventions for older adults with chronic diseases: a systematic review. H \& T, 6(2), 89-99 (2016).

[3] C. Williams, F. Mostashari, K. Mertz, E. Hogin, P. Atwal. From the office of the national coordinator: the strategy for advancing the exchange of health information. Health Affairs, 31(3), 527-536 (2012).

[4] W.M. Vollmer, A. Feldstein, D.H. Smith, J.P. Dubanoski, A. Waterbury, J.L. Schneider. Use of health information technology to improve medication adherence. AJMC, 17(12), 79-87 (2011).

[5] C.K.L. Or, B.T. Karsh. A systematic review of patient acceptance of consumer health information technology. J Am Med Inform Assn, 16(4), 550-560(2009).

[6] T.L. Webb, J. Joseph, L. Yardley, S. Michie. Using the internet to promote health behavior change: a systematic review and meta-analysis of the impact of theoretical basis, use of behavior change techniques, and mode of delivery on efficacy. JMIR, 12(1), 97-114 (2009).

[7] P. Sutcliffe, S. Martin, J. Sturt, J. Powell, F. Griffiths, A. Adams. Systematic review of communication technologies to promote access and engagement of young people with diabetes into healthcare. BMC Endocrine Disorders, 11(1), 1-11 (2011).

[8] China Internet Network Information Center. The 37th statistic report of Chinese Internet network development state (2016).

[9] E.B.C.M. Desroches, M.W. Painter, A.K. Jha. Health information technology in the united states, 2015: transition to a post-hitech world. Mathematica Policy Research Reports (2015).

[10] K.B. Eden, A.M. Totten, S.Z. Kassakian, P.N. Gorman, M.S. Mcdonagh, B. Devine. Barriers and facilitators to exchanging health information: a systematic review. Int J Med Inform, 88, 44-51 (2016).

[11] A.L. Hincapie, T.L. Warholak, A.C. Murcko, et al. Physicians' opinions of a health information exchange. JAMIA, 18(1), 60-65 (2011).

[12] D.C. Kaelber, D.W. Bates. Health information exchange and patient safety. J Biomed Inform, 40(6), S40-S45 (2007).

[13] D. Blumenthal. Launching hitech. NEJM, 362(362), 382-385 (2010). 
[14] G.J. Kuperman. Health-information exchange: why are we doing it, and what are we doing? JAMIA, 18(5), 678-682 (2011).

[15] O. El-Gayar, P. Timsina, N. Nawar, W. Eid. A systematic review of it for diabetes self-management: are we there yet? IJMI, 82(8), 637-652 (2013).

[16] K. Glanz, D.B. Bishop. The role of behavioral science theory in development and implementation of public health interventions. Annu Rev Public Health, 31, 399-418 (2010).

[17] T. Bickmore, T. Giorgino. Some novel aspects of health communication from a dialogue systems perspective. Aaai Fall Symposium on Dialogue Systems for Health Communication Washington Dc (2004).

[18] S. Barello, G. Graffigna, E. Vegni. The Challenges of Conceptualizing Patient Engagement in Health Care: A Lexicographic Literature Review. J Participat Med, 6 (2014).

[19] A. Coulter, S. Parsons, J. Askham. Where are the patients in decision-making about their own care? World Health Organization (2008).

[20] C.P. Lynch, J.S. Williams, K.J. Ruggiero, R.G. Knapp, L.E. Egede. Tablet-aided behavioral intervention effect on self-management skills (tablets) for diabetes. Trials, 17(1), 1-12 (2016).

[21] M.P. Gagnon, Nsangou ÉdithRomy, J. Paynegagnon, S. Grenier, C. Sicotte. Barriers and facilitators to implementing electronic prescription: a systematic review of user groups' perceptions. JAMIA, 21(3), 535-541 (2013).

[22] C. Dehay, H. Kennedy. Health Information Technology in the United States: Progress and Challenges Ahead 2014. Mathematica Policy Research Reports, 6(1), 119-140 (2014).

[23] Pew Research Center. Mobile Technology Fact Sheet. www.pewinternet.org/fact-sheets/mobile-technology-fact-sheet/. Accessed August 1, 2016.

[24] H. Martyn, L.M. Gallant. Over 50 and wired: web-based stakeholder communication. First Monday, 17(6) (2012).

[25] G.B. Winbush, L. Mcdougle, L. Labranche, S. Khan, S. Tolliver. Health empowerment technologies (het): building a web-based tool to empower older African-American patient-doctor relationships. Journal of Health Care for the Poor \& Underserved, 24(4), 106-117 (2013).

[26] D.J.C Carolina, J.G. Ros Wynand, S. Guus. The effects on health behavior and health outcomes of Internet-based asynchronous communication between health providers and patients with a chronic condition: a systematic review. JMIR, 16(1), 185-194 (2014).

[27] O.M. Ginsburg, M. Chowdhury, W. Wu, M.T.I. Chowdhury, B.C. Pal, R. Hasan, et al. An mhealth model to increase clinic attendance for breast symptoms in rural bangladesh: can bridging the digital divide help close the cancer divide? Oncologist, 19(2), 177-185 (2014).

[28] O. Bouhaddou, J. Bennett, T. Cromwell, et al. The Department of Veterans Affairs, Department of Defense, and Kaiser Permanente Nationwide Health Information Network exchange in San Diego: patient selection, consent, and identity matching. AMIA Symposium, 2011(2010), 135-143 (2011).

[29] C.S. Gadd, Y.X. Ho, C.M. Cala, et al. User perspectives on the usability of a regional health information exchange. JAMI A, 18(5), 711-716 (2011). 